\title{
Verbal Permission to Obtain Clinically Urgent Bio-Specimens for a Paediatric Biobank
}

\author{
Tamsin E Tarling ${ }^{1,4,5}$, Caron Strahlendorf ${ }^{1,4,5}$, Kirk R Schultz ${ }^{1,4,5}$, Ruth Milner ${ }^{1,4,5}$ and Suzanne M Vercauteren Ve,3, $^{*}$ \\ ${ }^{1}$ Department of Pediatrics, University of British Columbia, Vancouver, BC, Canada \\ ${ }^{2}$ Department of Pathology and Laboratory Medicine, University of British Columbia, Vancouver, BC, Canada \\ ${ }^{3}$ Division of Hematopathology, BC Children's Hospital, Vancouver, BC, Canada \\ ${ }^{4}$ Division of Hematology, Oncology, Bone Marrow Transplantation, BC Children's Hospital, Vancouver, BC, Canada \\ ${ }^{5}$ Child and Family Research Institute, Vancouver, BC, Canada
}

*Corresponding author: Suzanne M Vercauteren, BC Children's Hospital, Department of Hematopathology, 4480 Oak Street, Room 2K51, Vancouver BC, V6H 3N1, Canada, Tel: 604875 2939; Email: svercauteren2@cw.bc.ca

Rec date: Sep 05, 2014; Acc date: Oct 21, 2014; Pub date: Nov 04, 2014

Copyright: (c) 2014 Vercauteren SM, et al. This is an open-access article distributed under the terms of the Creative Commons Attribution License, which permits unrestricted use, distribution, and reproduction in any medium, provided the original author and source are credited.

\begin{abstract}
Objective: Consenting parents/guardians of paediatric patients suspected of a haematological malignancy for research is often a challenging process. However, without consent extra bone marrow cannot be taken for biobanking purposes. The Childhood Cancer and Blood Research (CCBR) BioBank was established to collect biospecimens from paediatric patients with haematological malignancies and blood disorders. Bio-specimens are also collected from patients with non-haematological malignancies that may have bone marrow involvement. Most patients present acutely with a potential diagnosis of childhood leukaemia, therefore there is considerable anxiety and distress at this time. Due to the acute nature of the presentation there is generally very little time between admission and the diagnostic procedure.
\end{abstract}

Method: To allow appropriate consideration to participate in biobanking a two stage consenting process was established with initial verbal permission for bio-specimen collection followed by full written consent at a later more appropriate time. A survey was conducted in the Haematology/Oncology/Blood and Marrow Transplant (Hem/Onc/ BMT) clinic of BC Children's Hospital to obtain opinions of patients and their families about biobanking in general and the consenting process used for the CCBR BioBank.

Results: Most eligible patients (93\%) consent to the CCBR BioBank. The majority of participants (71\%) preferred the two step process. Generally, participants understood and freely expressed their opinions about biobanking and the ethical issues associated. Participants felt helpful, honourable, hopeful or a combination of these having participated in the CCBR BioBank.

Conclusions: We found that verbal permission to collect a biobank specimen followed by formal written consent is an effective method of engaging informed patient participation in a biobank and is actually preferred by participants as a method of obtaining consent for biobanking in an urgent paediatric setting.

Terminology: For the purposes of this article, we have chosen to use the term bio-specimen or specimen when referring to the biological specimens that are obtained for biobanking. However, in our consent forms and in the survey that we conducted we use the term "sample" as we feel that this is easier for a lay person to understand. Therefore the terms bio-specimen, specimen and sample may be used interchangeably in this document. Additionally, when individuals are first approached in the hospital, we refer to them as patients. Once they have agreed to either participate in the CCBR BioBank or in the CCBR BioBank survey they become participants. The terms patient(s) or participant(s) refers to both the child and the parent or legal guardian.

Keywords: Biobank; Paediatric; Consent; Acute; Assent; Biospecimen; Ethical

\section{Introduction}

Biobanking is the process by which patient bio-specimens and clinical data are collected for research purposes. A recent survey addressed the definition of a biobank and concluded a biobank can be defined as "a facility for the collection, preservation, storage and supply of biological specimens and associated data, which follows standardized operating procedures and provides material for scientific and clinical use [1]". Advances in research technology have shown the increasing need for high quality preservation of bio-specimens with properly annotated data [2].

To obtain bio-specimens from patients for research purposes in an ethical manner, informed consent must be obtained as has been described in the Nuremburg Code [3], the Belmont Report [4], and the Declaration of Helsinki [5]. Valid informed consent is based on i) the participant agreeing to participate in the research voluntarily with no undue influence, ii) the participant being fully informed about the 
research they agree to participate in and iii) there being an element of on-going discussion regarding the individuals' participation in the research initiative [6]. Due to increasingly strict disclosure requirements by regulatory bodies, informed consent forms have become more and more complicated [7], despite the good intention to inform the patient of all possible outcomes.

Individuals consenting to participate in biobanking must fully understand their specimens and accompanying clinical data may be kept indefinitely and will be used in as yet undetermined research studies. Patients are often approached for biobank participation when they are first seen in a hospital; however they may not be in the right mind-set to decide whether to participate in a biobank in a truly informed manner. Procedures in which diagnostic and often the most valuable bio-specimens are obtained may occur within a short time frame, allowing little time to consent for the collection of biospecimens for biobanking purposes.

In the Paediatric setting, children under the age of majority cannot consent to their own participation in research. Parents or their legal guardians are designated to make this decision on their behalf. This becomes increasingly complicated in the field of biobanking where there is the potential for a child's bio-specimen to be stored for a considerable length of time, and well beyond the age when the child reaches the age of majority. The combination of stress, time pressures and a complex consent processes may further compromise the ability to make a true informed consent [8].

We investigated the use of a two-step consent process to collect biospecimens and annotated data for the CCBR BioBank from paediatric patients undergoing a bone marrow procedure for suspected haematological malignancies such as leukaemia. In support of this idea, a recent Permission to Contact strategy [9] hypothesizes that by breaking down the consent process improves patient engagement and thus enhances enrolment of patients into translational research programs.

We surveyed patients in the Hem/Onc/BMT clinic at BC Children's Hospital and their parents or legal guardians to determine their opinion of biobanking as well as their opinion of the two-step consenting process. We found that participants not only felt that biobank participation was honourable and helpful, but that it offered them some sense of hope. Additionally, the vast majority of participants preferred the two step consent process and felt well informed by the time the full consent process was completed.

\section{Methods}

\section{Consent Process}

The CCBR BioBank operates under the approval of the Children's and Women's Health Centre of BC (C\&W) Research Ethics Board (REB). To obtain consent from patients for biobanking, a two-step consent process was developed as summarized in figure 1. Patients undergoing a bone marrow procedure are informed by the physician about the BioBank. This usually happens within 24 hours of admission to the hospital. Patients are asked to give verbal permission (or verbal consent) to collect an additional 2-5 mls of bone marrow aspirate at the time of their procedure for biobanking purposes. Note, this consent only gives permission for the collection of additional biospecimens and not to biobanking at this time-point. If the patient agrees, a "witness of verbal consent form" is filled out by the physician or fellow (Figure 2). A welcome package including full consent and assent forms as well as a brochure about biobanking is given to the patient. The BioBank Coordinator will meet the patient within one month for full consent. Patients can withdraw at any time from the CCBR BioBank and they have two choices: a) to withdraw all existing bio-specimens and clinical data collected to date or b) to allow all existing bio-specimens and clinical data collected to date to remain in the CCBR BioBank with no further collection of bio-specimens or clinical information.

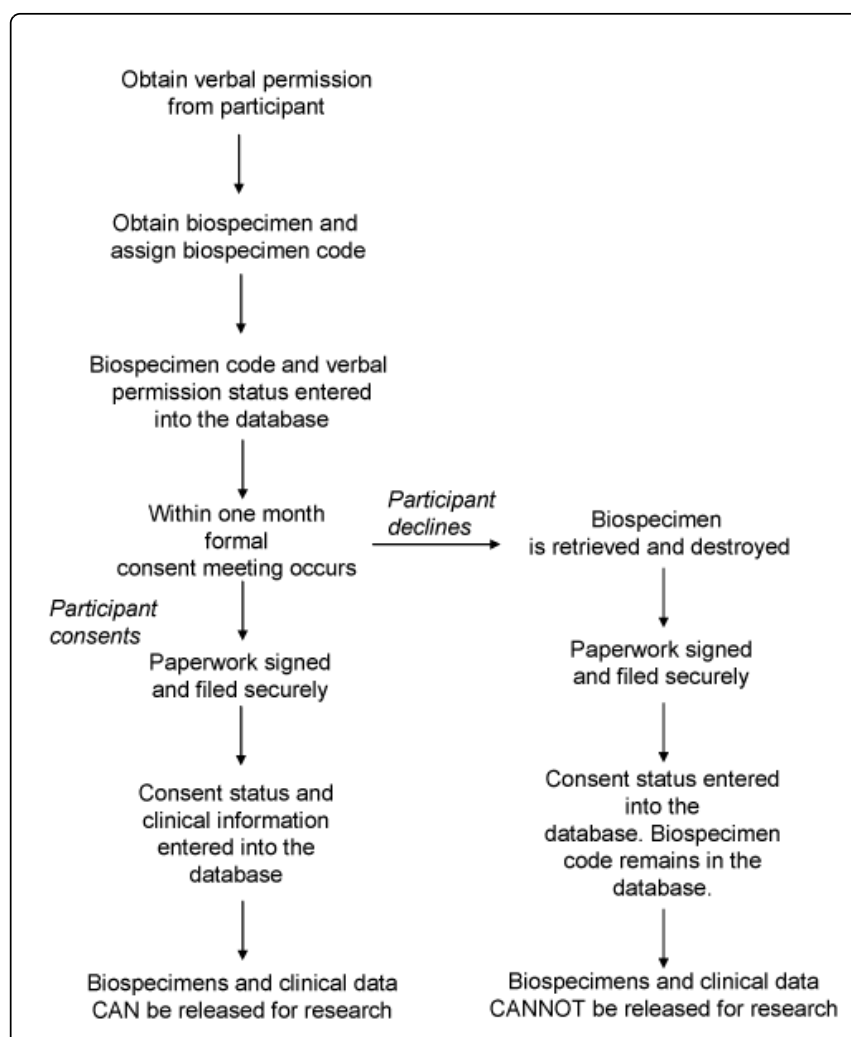

Figure 1: Verbal Permission Process for the CCBR BioBank. Process by which patients are recruited to participate in the CCBR BioBank.

\section{Bio-specimens and annotated data}

Bio-specimens are processed promptly and limited data is entered into the CCBR BioBank database, a customized version of the Advanced Tissue Management (ATiM), developed by the Canadian Tumor Repository (CTRNet) for biobanks (www.ctrnet.ca). The biospecimen is flagged in the database as "not-consented" and cannot be released for research purposes at this time. Once the full formal consent is completed and signed, clinical data and personal demographics are entered into the database; the specimens are then available for research purposes.

\section{Survey}

A written anonymous survey regarding the CCBR BioBank and the two step consenting process was approved by the C\&W REB. Surveys were distributed to patients in the outpatient $\mathrm{Hem} / \mathrm{Onc} / \mathrm{BMT}$ clinic as well as the inpatient ward of Hem/Onc/BMT at BC Children's Hospital between February 1 and July 31, 2013. 
The survey contained 27 questions, eight were general questions regarding biobanking and the remaining 19 were for CCBR BioBank participant's only and contained questions about the consent process. Completed surveys were deposited in drop boxes and were collected by the BioBank Coordinator weekly.

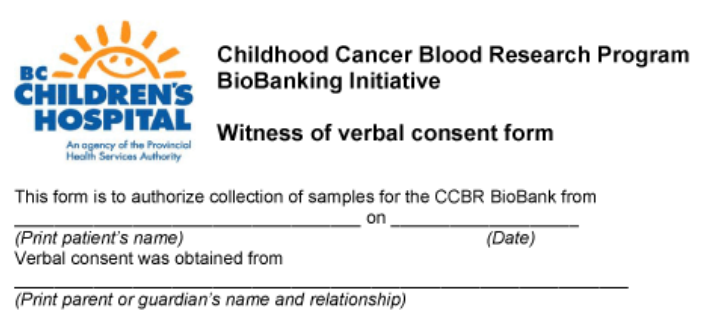

Instructions for fellows or physicians: Please read the statements below and Instructions for fellows or physicians: Please read the statements below and
initial in the relevant boxes to indicate that you discussed these issues with the patient.

patient.
I state that the above patient was informed of the CCBR BioBank and agreed to an extra 2-5 mls (approx 1 teaspoon) of bone marrow to be taken from their child for research purposes at the time of his/her bone marrow procedure. I have explained that this extra withdrawal will not affect their child.

I have explained that the BioBank Coordinator will meet with the parents in the next 1-2 weeks to discuss the CCBR BioBank in more detail and give them the opportunity to ask any questions they may have. At this time a formal consent form will be signed if applicable. they have met with form.

Should the patient be discharged from the hospital sooner than anticipated and before the BioBank Coordinator has managed to meet with them, the patient has agreed that the BioBank Coordinator can contact them by mail or telephone in order to follow up with the formal consent

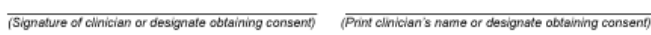

Please place addressograph sticker on this form. Attention: Please tube this form and the bone marrow requisition to HemPath (336) Please contact Tamsin Tarling, L6423, tamsin.tarling@cw.bc.ca with any questions

Figure 2: Witness of Verbal Consent Form. Documentation that the CCBR BioBank uses to record verbal permission for biospecimen collection

\section{Results}

\section{CCBR BioBank participation}

From September 2011 - September 2013, 154 patients were approached to participate in the CCBR BioBank; 95\% verbally consented to the collection of an additional bio-specimen at the time of the diagnostic procedure and $93 \%$ of those formally consented later. At this time $5 \%$ of patients had not yet been contacted for formal consent and 2\% declined formal consent. The BioBank code for the declined participants remains in the database (for statistical purposes) with a declined tag, but all identifying information is deleted. Biospecimens were removed from temporary storage and destroyed.

Formal consent was completed within a week of verbal consent for $27 \%$ of participants, within a month for $46 \%$ and in more than one month for $27 \%$ of participants. Patients that were not approached for formal consent within a month of verbal consent were patients that were discharged shortly after the bone marrow procedure as they had not been diagnosed with a malignancy and therefore did not have an early follow-up appointment at BC Children's Hospital. Ten patients (7\%) withdrew from the CCBR BioBank, all selecting a preference to keep the existing bio-specimens and clinical data in the CCBR
BioBank, but choosing to have no further bio-specimens or clinical data to be collected.

In July 2013, 50\% of CCBR BioBank participants had a diagnosis of acute leukaemia. Other diagnoses of participants include benign blood disorders (34\%), lymphoma with bone marrow involvement (1\%), lymphoma without bone marrow involvement $(4 \%)$, solid tumours with bone marrow involvement (2\%) and solid tumours without bone marrow involvement (9\%) (Figure 3). Bio-specimens collected were primarily bone marrow specimens. In a few cases where malignant cells were observed in the peripheral blood, cerebrospinal spinal fluid or other bodily fluids specimens, these fluids were also collected. The majority of specimens (56\%) were processed within four hours of collection with $27 \%$ being processed within 1 hour of collection.

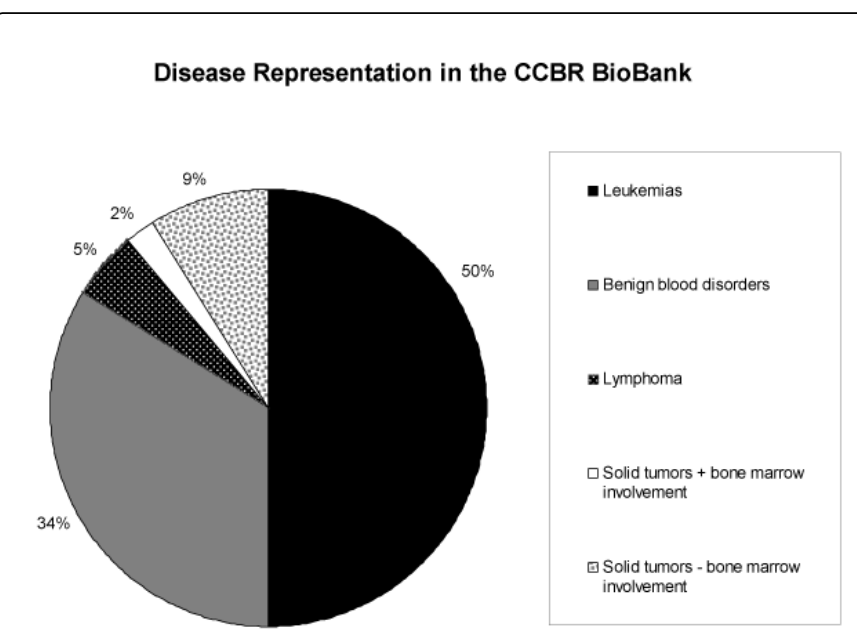

Figure 3: Disease Representation in the CCBR BioBank. Range of patients defined by their diagnosis which may undergo a diagnostic bone marrow procedure and therefore be approached to participate in the CCBR BioBank

\section{Survey}

Approximately 250 surveys were distributed in the outpatient clinic of $\mathrm{Hem} / \mathrm{Onc} / \mathrm{BMT}$ and the wards of Hem/Onc/BMT, 82 surveys (33\%) were returned. The surveys were completed by a parent or legal guardian in $96 \%$ of cases and $4 \%$ of surveys were completed by the patient themselves. Patient's age at the time of survey ranged from 0-5 years of age (34\%), 6-10 years of age and (18\%), 11-15 years of age (33\%), 16-21 years of age (15\%). Forty five per cent of survey participants were diagnosed within the last year. Disease types of all the survey participants included solid tumour (36\%), leukaemia (32\%), benign blood disorder (17\%), lymphoma (9\%), or unknown $(6 \%)$. Of the 82 surveys, $37 \%$ were from BioBank participants. Of all respondents, $39 \%$ had previously heard of a biobank while $55 \%$ had never heard of a biobank and $6 \%$ were unsure. Interestingly, $87 \%$ of respondents believed that participation in medical research via the donation of their bio-specimens would help to improve therapies and treatments for other patients with cancer or blood disorders (Figure 4) and $61 \%$ of respondents said they would be interested in finding out more about the CCBR BioBank.

Of the 30 BioBank participants who responded to the survey, $32 \%$ said that they were glad to have had the two step consent process and 
Citation: Tarling TE, Strahlendorf C, Schultz KR, Milner R, Vercauteren SM (2014) Verbal Permission to Obtain Clinically Urgent Bio-Specimens for a Paediatric Biobank. J Clinic Res Bioeth 5: 202. doi:10.4172/2155-9627.1000202

Page 4 of 7

that they found their decision making easier as a result, 39\% said that they were either glad or that they found their decision making easier as a result (Figure 5). Opinions regarding assent of CCBR BioBank participants were heterogeneous and included important (33\%), too overwhelming (27\%), too difficult (13\%) and "other" (27\%). However, further analysis showed a correlation between increasing age of child and increasing importance of assent (Figure 6).

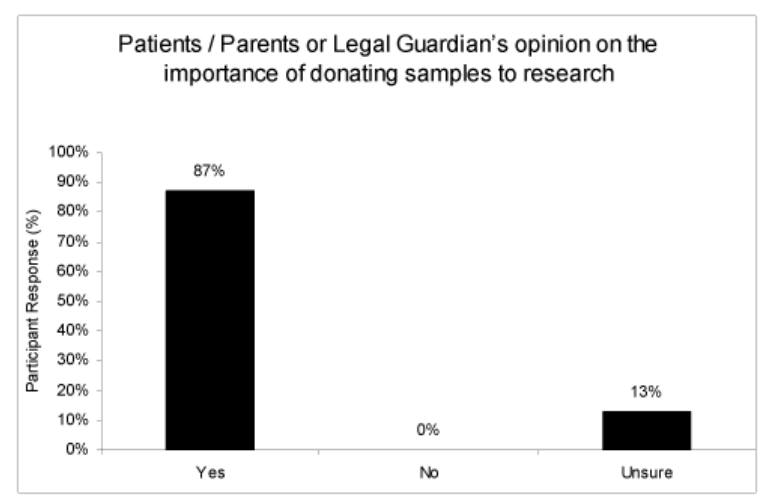

Figure 4: Patients / Parents of Legal Guardian's opinion on the importance of donating samples to research. CCBR BioBank Survey Participant's Response to the following question "Do you believe that your participation in medical research via the donation of YOUR samples will help to improve therapies and treatments for other patients with cancer or blood disorders?"

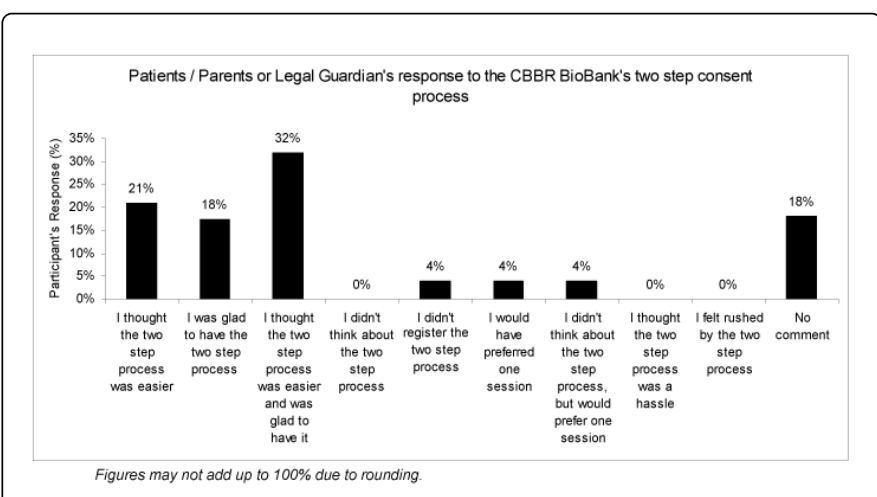

Figure 5: Patients / Parents of Legal Guardian's response to the CCBR BioBank's two step consent process. CCBR BioBank Survey Participant's Response to the following question "The CCBR BioBank intentionally set up a process by which your initial contact with the CCBR BioBank was via your doctor who asked you for a verbal consent only. The BioBank Coordinator then followed up with you in the weeks after your diagnosis for a signed formal consent. Please tick the boxes that you agree with regarding your impression of this 2 stage process."

Overall re-consenting at the age of majority was considered important by $57 \%$ of survey participants while $30 \%$ did not think it was important and 13\% were unsure. Figure 7 shows a further breakdown of these results and identifies the distribution of this opinion with age of patient at the time of completing the survey. More than $50 \%$ of CCBR BioBank participants in each age group felt that they would want to be approached for re-consent at the age of majority.

A brochure was given in the package at first contact; $77 \%$ of survey participants said that they read the brochure and of those $73 \%$ found it informative.

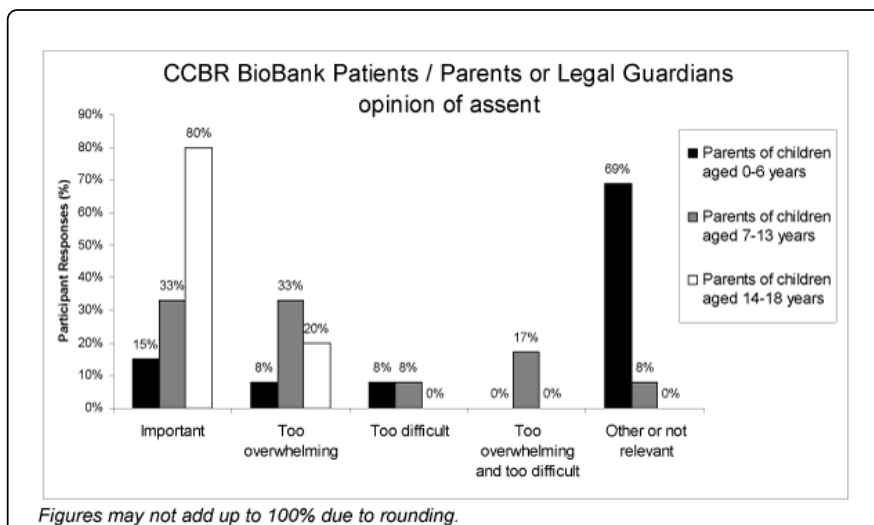

Figure 6: Patients / Parents of Legal Guardian's opinion of assent. CCBR BioBank Survey Participant's Response to the following question, "The CCBR BioBank feels that it is important to assent children over the age of 7 years. We think that as informed human beings they have the right to know about their involvement in research. Please tick the boxes below to express your opinion of the assent process". Age groups represent the current age of the patient at the time of being asked the above question.

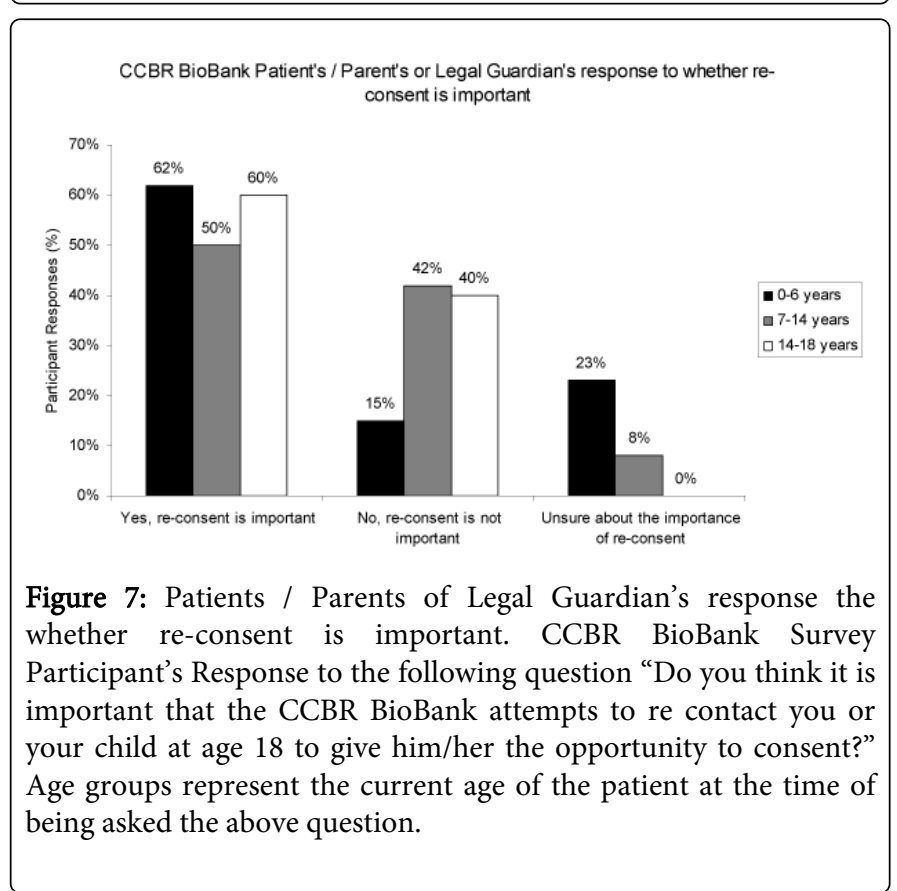

We also asked CCBR BioBank participants what their understanding was in regard to the clinical information and the types of specimens that could be taken for the CCBR BioBank. Most participants were aware that bone marrow was being collected (77\%), and $67 \%$ participants thought that blood was or could be collected. A small number (27\% participants) remembered that CSF could be 
collected, $27 \%$ of participants remembered that stem cells could be collected and $13 \%$ remembered that leukopheresis product could also be collected. None of the participants checked the "yes" box for urine or stool collections which is correct, although $10 \%$ of participants thought that they had given permission for these specimens to be collected should a situation arise. Currently these two specimens are not collected for the CCBR BioBank. In terms of clinical data or personally identifying data, $40 \%$ of participants were aware that clinical data was collected and $33 \%$ of participants were aware that personal identifiers were collected for the CCBR BioBank (Figure 8).

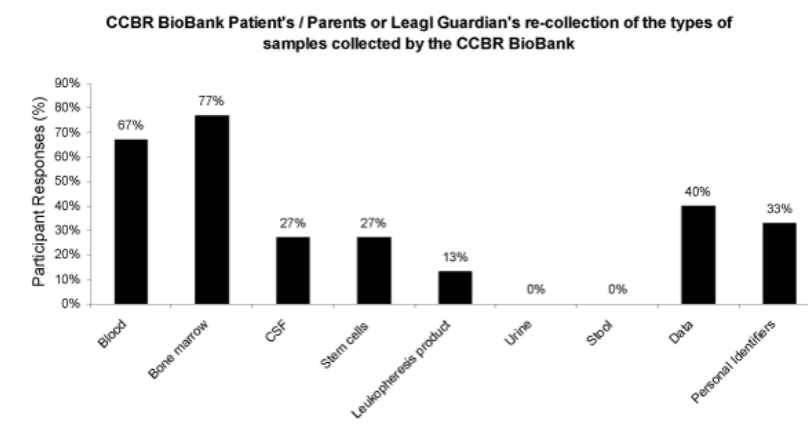

Figures do not add up to $100 \%$ due to participants checking multiple boxes. Percentage is calculated as number of responses for each specimen type $\div$ number of participant responses $\times 100 \%$.

Figure 8: Patients / Parents of Legal Guardian's re-collection of the types of samples collected by the CCBR BioBank . CCBR BioBank Survey Participant's Response to the following question "If you agreed to participate in the CCBR BioBank, what is your understanding of the samples and electronic information currently being stored? Please check the appropriate boxes"

Concerns regarding the consent form were expressed by $10 \%$ of CCBR BioBank participants who completed the survey and the concerns were mostly regarding privacy and whether collection of additional specimens can harm the health of the child.

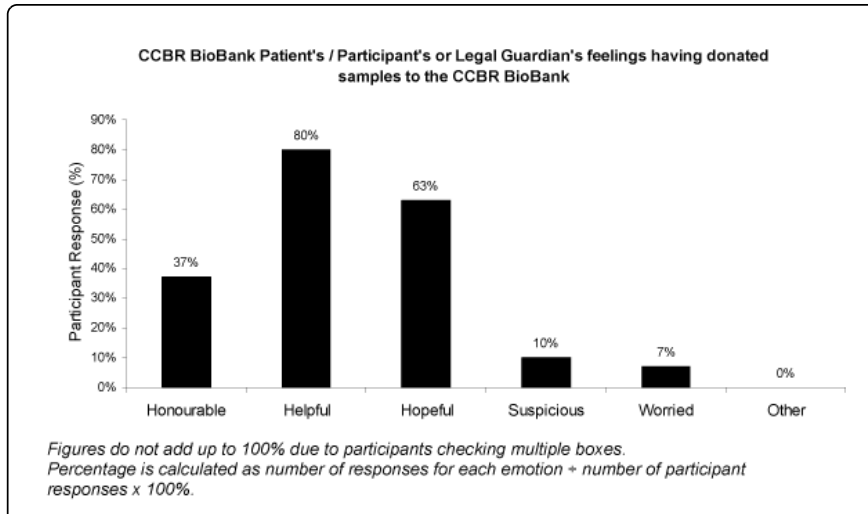

Figure 9: Patients / Parents of Legal Guardian's feelings having donated samples to the CCBR BioBank. CCBR BioBank Survey Participant's Response to the following question "How do you feel now that you have given your sample to the CCBR BioBank for research purposes? Please check the boxes that represent your feelings below". Participants were able to check more than one box, the figure shows the number of survey participants who checked each emotion.

Twenty seven per cent of BioBank participants who responded to the survey felt a right to know relevant discoveries through research and their bio-specimen donation, $13 \%$ would like to have access to research publications and $27 \%$ felt a right to know relevant discoveries through research and their bio-specimen donation and would like access to research publications. Only $27 \%$ of respondents said they were happy to have contributed to research and would not want to know any more about research findings, $6 \%$ had no opinion. Overall these results suggest that $67 \%$ of CCBR BioBank participants would be interested in finding out about the research they have contributed to.

Finally, we addressed the feelings participants' experience, having given their specimens to the CCBR BioBank. Overwhelmingly, participants feel either hopeful, honorable or helpful or a combination of these. Figure 9 shows the number of participants who checked each box, participants were able to check more than one emotion. A small number of respondents have feelings of worry or suspicion.

\section{Discussion}

Typically paediatric patients with suspected acute leukaemia are admitted to the hospital in an emergency setting. Within a short time frame, (usually 12-24 hours) they will undergo a bone marrow procedure for diagnostic purposes. In this acute situation, parents or legal guardians of these patients have to process a significant amount of information, address emotional challenges and sign several consent forms including those for diagnostic procedures and immediate treatment. In 2007, a public engagement event, the BC Biobank Deliberation was conducted and one of the key messages from these deliberations was that "special provision should be made for individuals who are just about to receive surgery, as they may not be in a position to digest important information and thus not able to provide an informed consent [10]". We therefore developed a two-step consent process in which the permission to collect an additional specimen for biobanking purposes is obtained by the primary caregiver followed by a formal consent by the BioBank Coordinator after the procedure has taken place and a diagnosis has been made. 
The vast majority of patients approached by their primary caregiver give permission to collect additional bio-specimens and proceed to sign the formal consent later. We believe that this high recruitment rate is a reflection of consent process we have developed. However, there are disadvantages to the two step consent process. First, on rare occasions a patient did give verbal permission to collect a biospecimen but later did not remember this. Although this is worrisome the number of participants that it relates to is minimal and we believe the remainder are well informed. It has been commented upon that patients have been consented for other studies at times of great emotional stress and did not remember having signed a consent form. The second disadvantage to the two step consent process is that researchers do not have access to fresh cells as the processed cells must be stored frozen, until formal consent is obtained.

During consent conversations, we have observed that patients express a willingness to participate in biobanking with the hope of improving survival and quality of life for other patients. Our survey verifies this, as $87 \%$ of survey participants thought that donating patient specimens to research would help improve medical treatment in the future. Additionally, the majority of CCBR BioBank participants who completed our survey said that they felt hopeful and/or helpful and/or honorable as a result of donating bio-specimens to the CCBR BioBank. We interpret these results to reflect a wish to be engaged in research and a trust or hope that research can make a difference to future medical care. Increasingly, research supports the concept that patients are interested in participating in research with the hope of improving treatments and therapies or "finding a cure" for others in the future [11].

The survey we developed covered some general points whose purpose was to determine how much CCBR BioBank participants understood the consent form. The vast majority of participants said that they understood the consent form and that they knew samples would only be collected at the time of a clinical procedure. Additionally, most participants were able to recall the specimens that the CCBR BioBank collects accurately.

Our survey also addressed three ethical issues which are pertinent to paediatric biobanking; 1) The importance of obtaining assent from children, 2) The importance of re-consenting young adults when they reach the age of majority and 3) The return of research results or incidental findings.

In regard to assent, CCBR BioBank participants showed a trend of increasing importance with increasing age. Interestingly, there was an equal distribution of families who thought that assenting between the ages of 7 and 14 years was either important or too overwhelming. Our survey did not clearly distinguish between whether it was more important to engage the child in the discussion of participation in a biobank versus the actual documentation of the assent.

The majority of our survey participants thought it was important to re-consent at the age of 18 . Logistically and financially, this may be a challenge for biobanks, but it is something that paediatric biobanks should be prepared for. A recent survey of Canadian, adult leukaemia patients addressed the issue of re-consent, approximately $50 \%$ of adults felt that re-consent was more respectful and allowed them to be more involved in research [12]. However, it should be noted that the emphasis of adult re-consent is different to that in a paediatric setting. We are currently performing another, more extensive paediatric study which suggests re-consent is important to paediatric patients.
The return of research results or incidental findings is a difficult issue especially when it pertains to the paediatric population. The previously mentioned biobank deliberation reported that there was "persistent disagreement on the return of research results and ownership [10]". The TCPS 2 states that "researchers have an obligation to disclose to the participant any material incidental findings discovered in the course of research [6]". A study from 2013 found that $66 \%$ of adult cancer patients would prefer it if incidental findings were reported to both them and their doctor [12]. Similarly, the majority of our survey participants preferred the return of results, but it should be noted that the participants of our survey were not well informed on the issue of the return of results and the potential positive and negative consequences of returning research results.

In summary the majority of paediatric patients with cancer or blood related disorders are happy to participate in biobanking and have positive, altruistic views regarding the banking of paediatric biospecimens for use in future medical research.

A two-step consent process for biobank participation for paediatric patients undergoing an acute diagnostic procedure is preferred and likely augments the understanding of the consent form regarding biobanking.

We recognize the limitations of our survey, including the low participation rate and the bias towards English speaking participants as well as participants who have considerable experience with the BC health care system.

\section{Acknowledgements}

We would like to thank clinical staff of the Division of Hematology/ Oncology/Bone marrow Transplant Division at BC Children's Hospital for obtaining verbal consent for the CCBR BioBank and the Childhood Cancer and Blood Research Program of the Child and Family Research Institute for initial funding.

We would also like to thank our collaborators, the Canadian Tumour Repository Network (CTRNet) and the University of British Columbia, Office for Biobank Education and Research (OBER) for their advice and support of this project.

\section{References}

1. Hewitt R, Watson P (2013) Defining biobank. Biopreserv Biobank 11: 309-315.

2. Filocamo M, Baldo C, Goldwurm S, Renieri A, Angelini C, et al. (2013) Telethon Network of Genetic Biobanks a key service for diagnosis and research on rare diseases. Orphanet J Rare Dis 30: 129.

3. The Nuremberg Code (1947) US, National Institute of Health.

4. The Belmont Report (1979) Ethical Principles and Guidelines for the Protection of Human Subjects of Research.

5. Declaration of Helsinki (1964) Ethical Principles for Medical Research Involving Human Subjects. World Medical Association.

6. (2010) Tri Council Policy Statement (2nd Edn) Ethical Conduct for Research Involving Humans. Panel of Research Ethics, Government of Canada.

7. Presidential Bioethics Commission Issues Report on Clinical Trials Research in Developing Countries (2001) National Bioethics advisory commission.

8. McMurter B, Parker L, Fraser RB, Fergall Magee J, Kozancyzn C, et al. (2011) Parental views on tissue banking in pediatric oncology patients. Pediatr Blood Cancer. 57: 1217-1221. 
Citation: Tarling TE, Strahlendorf C, Schultz KR, Milner R, Vercauteren SM (2014) Verbal Permission to Obtain Clinically Urgent Bio-Specimens for a Paediatric Biobank. J Clinic Res Bioeth 5: 202. doi:10.4172/2155-9627.1000202

Page 7 of 7

9. Cheah S, O’Donoghue S, Daudt H, Dee S, LeBalnc J (2013) Permission to contact (PTC)--a strategy to enhance patient engagement in translational research. Biopreser Biobank 11: 245-252.

10. Doherty KC, Burgess MM (2009) Engaging the public on biobanks: outcomes of the BC biobank deliberation. Public Health Genomics 12 203-215.
11. Braun KL, Tsark JU, Powers A, Croom K, Kim R, et al. (2014) Cancer patient perceptions about biobanking and preferred timing of consent. Biopreserv Biobank 12: 106-112.

12. Master Z, Claudio JO, Rachul C, Wang JCY, Minden MD, et al. (2013) Cancer patient perceptions on the ethical and legal issues related to biobanking. BMC Med Genomics 6: 8 . 